\title{
CONVERGENCE DELAY IN OSPF: A BRIEF REVIEW
}

\author{
Pertik Garg ${ }^{1} \&$ Anuj Kumar Gupta ${ }^{2}$
}

Abstract- Fast convergence in OSPF is very important for widespread deployment of real time applications. The present study improves the OSPF performance convergence by providing back up path, improving OSPF routing algorithm, in case of failure, and makes it a preferred choice for today's network designers.

Keywords - OSPF, Router, Packet loss, Convergence time.

\section{INTRODUCTION}

\subsection{Open Shortest Path First (OSPF)}

Open Shortest Path First (OSPF) [1] is a link state routing protocol (LSRP) that uses the Shortest Path First (SPF) network communication algorithm (Dijkstra's algorithm) to calculate the shortest connection path between known devices.

\subsection{How OSPF works}

- $\quad$ Routers in exchange link-state data start the process.

- $\quad$ Each router stores the link-state information in memory using a structure named the topology table or topology database.

- $\quad$ The router processes all data in the topology table and makes use of the Dijkstra algorithm to determine all routes to all networks, as well as the least-cost routes.

- $\quad$ All this information is stored in the SFP tree, identifying preferred and secondary routes.

- $\quad$ The routing information is propagated to the routing table as shown in figure 1.

When designing our OSPF network, the two main factors we have to work with are areas and how they fit within an AS. Areas are functional areas of our network, perhaps a building or the floor of a building, and Autonomous Systems are collections of areas, which typically are our entire network as shown in figure 2.

The overall OSPF network is divided into groups called areas, whereas all routers in an organization are probably part of a single AS. The area is defined as a logical division of the AS, broken up into contiguous sections of the IP network.

In other words, we break the area along groups of subnets that can be grouped together with a single routing entry. In a typical large network, an area may consist of 30 to 40 routers.
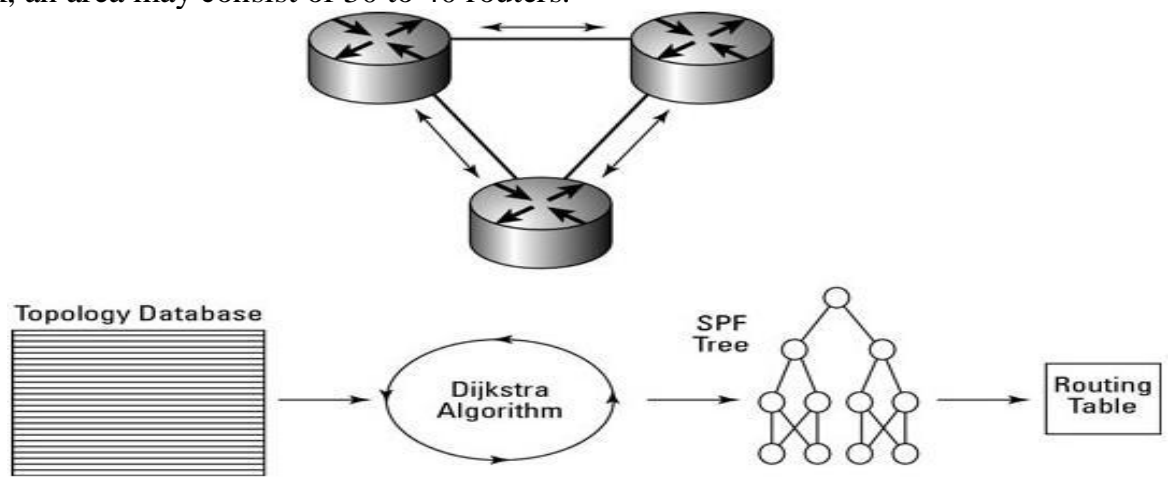

Figure 1. SPF tree generate from topology table

${ }^{1}$ Department of Computer Science Engineering, Punjab Technical University, Jalandhar, India

${ }^{2}$ Department of Computer Science Engineering, CGC, Landran, Punjab, India 


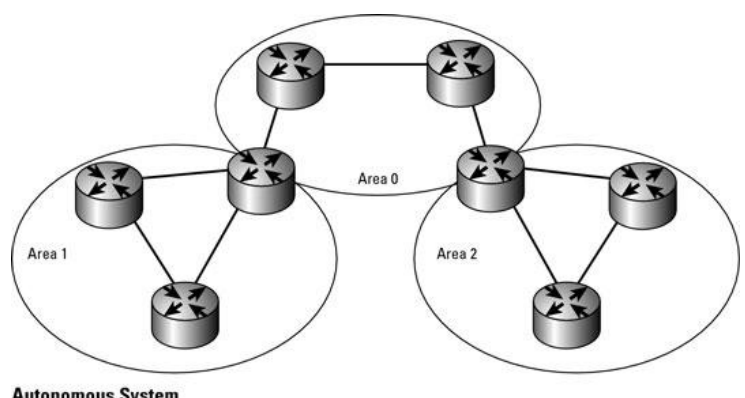

Figure 2. Link State Routing Protocol

\subsection{Growth in Industry}

OSPF [2] was developed by the Internet Engineering Task Force (IETF). Compared with IGRP or EIGRP, OSPF features higher efficiency, better interoperability, and faster convergence, and supports more protocol extensions. Devices from many vendors support OSPF. OSPF has been widely used in large scale networks and is the IGP recommended by the IETF Therefore, many networks running IGRP/EIGRP need to migrate to OSPF for network expansion and adjustment

Suppose we have an OSPF network, and our company merges or acquires or needs to communicate with another that also runs OSPF. The solution is to use multiple OSPF Autonomous Systems (routing domains), and run some other protocol (BGP?) between the Autonomous Systems (AS's).

This may also be useful in a multi-national corporate network. Suppose our company has networks in the North America, Europe, Asia, Australia, and perhaps those other continents too. Are they under common administration? One suspects not. So controlling growth, addressing, and so on centrally could be a challenge. By using OSPF we can find the solution The U.S. Post Office is putting in 38,000 Cisco routers. Note that if we use our rules of thumb, 38,000 divided by 100 routers says we'd need 380 areas. Alternatives include multiple OSPF AS's, and other protocols (like EIGRP). We required healthy route summarization in such a network. Note that since OSPF only allows us to summarize at ABR's, we can only have one level of summarization. With 38,000 routers, we might well want to summarize at the regional, state, and portion of state levels. That can't be done with pure OSPF.

\section{LITERATURE REVIEW}

Norihito Fujita et al. (2001)[3] suggested two schemes for efficient pre-computation of multiple paths: (i) adaptive precomputation based on the current $\mathrm{CPU}$ load to reduce the convergence time for the first bootstrapping, and (ii) a virtual area partitioning (VAP) algorithm to reduce the update time of the multiple candidate paths

ZHOU Haiju, et al. (2003) [4] analyzes the local congestion issue of network owing to traffic aggregation and improved OSPF and brought forward cost adaptive OSPF (CA-OSPF)

Fernando Barreto, et al. (2008) [5] proposes a proactive approach of Fast Recovery Paths to aid OSPF bypass failures. According to their evaluation paths are shorter than other similar approaches and they significantly reduce the packet loss rate during the OSPF convergence period caused by a failure.

M Goyal, et al. (2009)[6] discussed that large hold time value limits the frequency of routing table calculations but also causes large delays in convergence to the topology change. They analyze the ability of different hold time schemes to limit the frequency of routing table calculations under continuous link state advertisements (LSA) arrivals starting with a small value for the hold time.

Vincenzo Eramo, et al. (2009) [7] analyzes intra-domain routing protocols improvements to support new features required by real time services. They propose a new multi-path dynamic algorithm which uses multipath information to make a fast determination about the new shortest paths when a link failure occurs, reducing this way the network re-convergence time Xuezhi Jiang, et al, (2009) [8] presented the paper on Improving IGP Convergence through Distributed OSPF in Scalable Router; they propose a distributed OSPF (DOSPF) scheme to schedule routing computation through self-adaptively adjusting SPT waiting time.

Jaewon Kang, et al, (2010) [9] proposes an Adaptive Link Establishment (ALE) scheme that creates a link based on its stability in terms of connectivity and channel condition. The key characteristic of the ALE scheme is that it dynamically controls the latency of a new link creation by either accepting or dropping incoming Hello messages without generating additional control packets on the wireless channel. In addition, the ALE scheme is fully compatible with the legacy OSPF routing protocol.

M. Haider, et al, (2011) [10] presented a new routing table calculation scheme for OSPF routing protocol to better serve realtime applications. The proposed scheme focus on speeding up OSPF networks convergence time by optimizing the scheduling of routing table calculations using computational intelligence technique.

John Dwyer, Hetal Jasani, (2012) [11] analysis of convergence delay caused by link failures in Autonomous Systems. They present that due to the importance of computers and network resources, many organizations implement redundant links to allow failover in the event of a link failure. When this failover occurs, the network must converge before traffic will be able to pass to and from the network segment that incurred a link failure. The time that is required for the network to converge is referred to as convergence delay. 
S.U. Malik, et al, (2012) [12] proposed a novel method to compute the intra-area convergence time of open shortest path first (OSPF) based networks in the presence of designated routers (DRs) on Ethernet and non-broadcast multi-access segments.

\section{CONCLUSION}

The proposed work will improve the functionalities of OSPF. Improved algorithm can quickly respond to the topology change and reduce the convergence time by providing back up path which is already stored in routing table before the failover occurs. This algorithm reduces the convergence delay caused by link failures in OSPF.

By reviewing the various papers on OSPF we conclude that if back up path will be give to OSPF its performance will be improved by reduction of convergence delay.

\section{REFERENCE}

[1] Karamjeet Kaur, Sukhjeet Singh and Rahul Malhotra(2012), "Design of Open Shortest Path First Protocol -A Link State Protocol using OPNET Modular", International Journal of Computer Science and Mobile Computing (IJCSMC), Vol. 1, Issue 1, pg. 21 - 31.

[2] Peter J. Welcher, "Introducing OSPF".

[3] Norihito Fujita and Atsushi Iwata (2001). Adaptive and Efficient Multiple Path Pre-computation for QoS Routing Protocols, 2001 IEEE.

[4] ZHOU Haijun, PAN Jin, SHEN Pubing (2003). Cost Adaptive OSPF, Fifth International Conference on Computational Intelligence and Multimedia Applications (ICCIMA'03), 2003 IEEE.

[5] Fernando Barr, Emílio C. G. Wille, Luiz Nacamura Junior (2008). Fast Recovery Paths: Reducing Packet Loss Rates during IP Rou ting Convergence. The Fourth Advanced International Conference on Telecommunications, 2008 IEEE.

[6] MGoyal, M Soperi, H Hosseini (2009). Analyzing the Hold Time Schemes to Limit the Routing Table Calculations in OSPF Protocol 2009 International Conference on Advanced Information Networking and Applications, 2009 IEEE.

[7] Vincenzo Eramo, Marco Listanti, and Antonio Cianfrani (2008). Design and Evaluation of a New Multi-Path Incremental Routing Algorithm on Software Routers. IEEE Transactions On Network And Service Management, Vol. 5, No. 4, December 2008,2008 IEEE.

[8] Xuezhi Jiang, Mingwei Xu, Qi Li, Lingtao Pan (2009). Improving IGP Convergence through Distributed OSPF in Scalable Router. 2009 11th IEEE International Conference on High Performance Computing and Communications, 2009 IEEE

[9] Jaewon Kang, Mariusz A. Fecko and Sunil Samtani (2010). ALE: Adaptive Link Establishment in OSPF Wireless Ad-Hoc Networks The 2010 Military Communications Conference ,2010 IEEE.

[10] M. Haider, Mohd Zahid M Soperi, Kamalrulnizam Abu Bakar (2011). Comparison of Intelligent Schemes for Scheduling OSPF Routing Table Calculation, 2011 IEEE.

[11] John Dwyer, Hetal Jasani(2012). An Analysis of Convergence Delay Caused by Link Failures in Autonomous Systems, 2012 IEEE.

[12] S.U. Malik, S.K. Srinivasan and S.U. Khan. (2012). Convergence time analysis of open shortest path first routing protocol in internet scale networks. Electronics Letters, 13th September 2012 Vol. 48 No. 19 